\title{
Influence of the Transverse Component of the Interplanetary Magnetic Field on the Size of the Auroral Oval
}

\author{
Hitoshi NAKAI \\ Makino High School/Physics, Hirakata, Osaka, Japan \\ (Received January 9, 1987; Revised June 23, 1987)
}

\begin{abstract}
The auroral boundary index calculated by GUSSENHOVEN et al. (1983) on the basis of precipitating electron data is used to examine the response of the auroral oval in the midnight sector to variations of the $B_{y}$ component of the interplanetary magnetic field (IMF). It is found that the size of the auroral oval with positive $B_{y}$ is larger (smaller) than that with negative $B_{y}$ when the earth's dipole tilt angle is negative (positive). This $B_{y}$ dependence of the auroral oval occurs in the same way in both the northern and southern hemispheres. It is also found that when the magnitude of the transverse component of the IMF is fixed, the largest oval occurs in association with the IMF orientating to $\Theta=11.3,0.7$, and $-20.5^{\circ}$ when the tilt angle of the geomagnetic dipole $(T)$ is in the ranges of $T<-10,-10 \leq T<10$, and $T \geq 10^{\circ}$ respectively. Here, $\Theta$ indicates the angle measured anticlockwise from the south to the transverse component of the IMF viewed from the sun. The results of data analyses can be explained in terms of the dawnside preference of the dayside magnetic reconnection.
\end{abstract}

\section{Introduction}

By examining variations in geomagnetic activity recorded by geomagnetic indices such as the $A E$ index, it has been recognized that solar wind energy is injected into the magnetosphere not only when the interplanetary magnetic field (IMF) points south but when it has other orientations as well. The influence of the $B_{y}$ component of the IMF on geomagnetic activity and on plasma flow at high latitudes has been reported by many authors (e.g., HEPPNER, 1972; FRIIS-CHRISTENSEN et al., 1972, 1985; PoteMra and SAFLEKOS, 1979; MATSUSHITA and XU, 1982). In a series of papers, AOKI (1977), HAKAMADA et al. (1980), and MURAYAMA et al. (1980) showed that the $A L$ index depends negatively (positively) on the IMF $B_{y}$ when the tilt angle of the earth's dipole axis is positive (negative). Recently, REIFF et al. (1981), WYGANT et al. (1983), and DOYLE and BURKE (1983) showed that the magnitude of the observed polar cap potential drop can be reproduced by theoretical equations deduced from the reconnection hypothesis. Unfortunately, however, these works do not address the question of the tilt angle dependence of the IMF $B_{y}$ effect.

Unveiling the quantitative dependence of the IMF $B_{y}$ on magnetospheric processes will put meaningful constraints on theoretical models of dayside reconnec- 
tion. Murayama et al. (1980) suggested that a more intense westward auroral electrojet flows when the reconnection takes place at the dawnside than at the duskside of the polar cusp. More recently HEELIS (1984) used data of ion convection velocities obtained by $A E-C$ to explain the dawnward displacement of the convection "throat", a sunward-antisunward convection reversal zone at the polar cap boundary, in terms of a "preferred" dawnside reconnection (CROOKER et al., 1985). The cause of the dawnside preference, however, is not sufficiently clear at present. Besides, these previous papers are based on observations at northern high latitudes only. Thus there still remains the important question of whether the $B_{y}$ effect results in a global modulation or a north-south asymmetry of geomagnetic activity.

The size of the auroral oval is one important measure of the relationship between solar wind parameters and geomagnetic activity (HOLZWORTH and MENG, 1975; KAMIDE and WINNINGHAM, 1977; HARDY et al., 1981; NAKAI and KAMIDE, 1983). In particular, the lowest latitudes of the equatorward auroral boundary in each oval expansion correlate very well with the IMF $B_{z}$ component (PIKE and DANDEKAR, 1979; NAKAI et al., 1986a, b). The purpose of the present paper is to investigate the influence of $B_{y}$ on the maximum size of the auroral oval by utilizing the auroral boundary index presented by GUSSENHOVEN et al. (1982). This paper first investigates whether the northern and southern ovals respond to the IMF $B_{y}$ in the same sense or in a opposite sense. Second, it examines which component, $B_{x}$ or $B_{y}$, is more important in determining the oval size. In addition, we discuss the possible causes of $B_{y}$ effects, and the dayside reconnection rate in the light of some of the theoretical predictions.

\section{Procedure of Data Analysis}

GUSSENHOVEN et al. $(1982,1983)$ utilized DMSP $F 2$ and $F 4$ precipitating electron data for 1978 to determine the corrected geomagnetic latitudes of the equatorward boundaries of the auroral ovals in both hemispheres. They prepared a data set containing latitudes of measured boundaries and values of the auroral boundary index derived by mapping the measured boundaries to geomagnetic midnight using statistical formula.

The auroral oval expands generally in association with an increase in the southward component of the IMF, and it contracts as the IMF turns to the north (e.g., KAMIDE and WINNINGHAM, 1977; HARDY et al., 1981; NAKAI and KAMIDE, 1983). By utilizing the auroral boundary index, NAKAI et al. $(1986 \mathrm{a}, \mathrm{b})$ showed that associated with the southward or northward turning of the IMF, the auroral oval expands and contracts with time scales of 45 minutes and 8 hours, respectively. The long time-scales of the oval contraction make correlation coefficients between the oval size and the solar wind parameters rather low. Indeed, the "well expanded" oval is often observed even during periods of the northward IMF (NAKAI and KAMIDE, 1983). On the other hand, since the auroral oval can expands more rapidly than it can contract (PIKE and DANDEKAR, 1979), the equatorward boundary of the auroral oval seems to correlate well with the southward $B_{z}$ (HARDY et al., 1981). As noted in the 
Introduction, PIKE and DANDEKAR (1979) and NAKAI et al. (1986a, b) have demonstrated that the lowest latitude of the equatorward boundary highly correlates with the IMF $B_{z}$.

Considering the expansion time-scale of 45 minutes, 1 hour running averages of the IMF $B_{z}$ for 1978 are used. Our data set consists of the negative peaks of the hourly averaged $B_{z}$ values for which there were auroral boundary index values available within 1 hour. To avoid the possible influences of preceding substorm activity, we have excluded the negative peaks before which a greater southward excursion of the IMF occurred within less than 6 hours. Note also that the present data set does not include data during the period of the northward IMF.

Figure 1 shows variations of the 1 hour running averages of $B_{z}$ (top panel), the $A L$ index (middle panel), and the auroral boundary index on May 11, 1978 (bottom panel). Solid and open circles in the bottom panel indicate the auroral boundary index derived from measured boundaries in the northern and southern hemispheres, respectively. The minimum peaks of $B_{z}$ occurred near 0350 UT, 1320 UT, 1700 UT, and $2250 \mathrm{UT}$. The events at $1320 \mathrm{UT}$ and $2250 \mathrm{UT}$ are not included in our data set because there were smaller $B_{z}$ within 6 hours before the observations. After the minimum $B_{z}$ occurred at 0350 UT the DMSP satellite crossed the northern oval at 0407 UT and the southern oval at 0438 UT, when the auroral boundary index values were 54.1 and 53.3 CGL, respectively. The auroral oval contracted slowly until about $1400 \mathrm{UT}$, and the slightly expanded oval located at 58.9 CGL was observed at about 1746 UT. In association with these two oval expansions, two normal substorms occurred, as one can see in the $A L$ index. However, substorms, except for exceptionally huge one, seem not to significantly influence the oval size (NAKAl et al., 1986b). Therefore in the present paper we do not discuss further on the effect of substorms.

Searching the solar wind parameters and the auroral boundary index for 1978, we found 239 events. To avoid influence from intense magnetic storms, we excluded 36 events for which the transverse component of the $\operatorname{IMF}\left(B_{\mathrm{t}}\right)$ is greater than $10 \mathrm{nT}$. Finally, 203 records are available for the following analysis. Among them are 12 events in which values of the auroral boundary index were obtained in both hemispheres within 1 hour of the occurrence time of the corresponding minimum $B_{z}$, for example at 0350 UT on May 11, 1978. These pairs of data are regarded as independent of one another.

\subsection{Dependence of the auroral boundary index on the IMF $B_{z}$}

Values of the auroral boundary index $(\Lambda)$ are plotted as a function of $B_{z} V$ in Fig. 2 , where $V / 400$ stands for the solar wind speed normalized by $400 \mathrm{~km} / \mathrm{s}$. Solid and open circles indicate the auroral boundary indices observed in the northern and southern hemispheres, respectively. A solid line in the figure indicates a regression line expressed by

$$
\Lambda=65.5+1.02 B_{z} V / 400,
$$




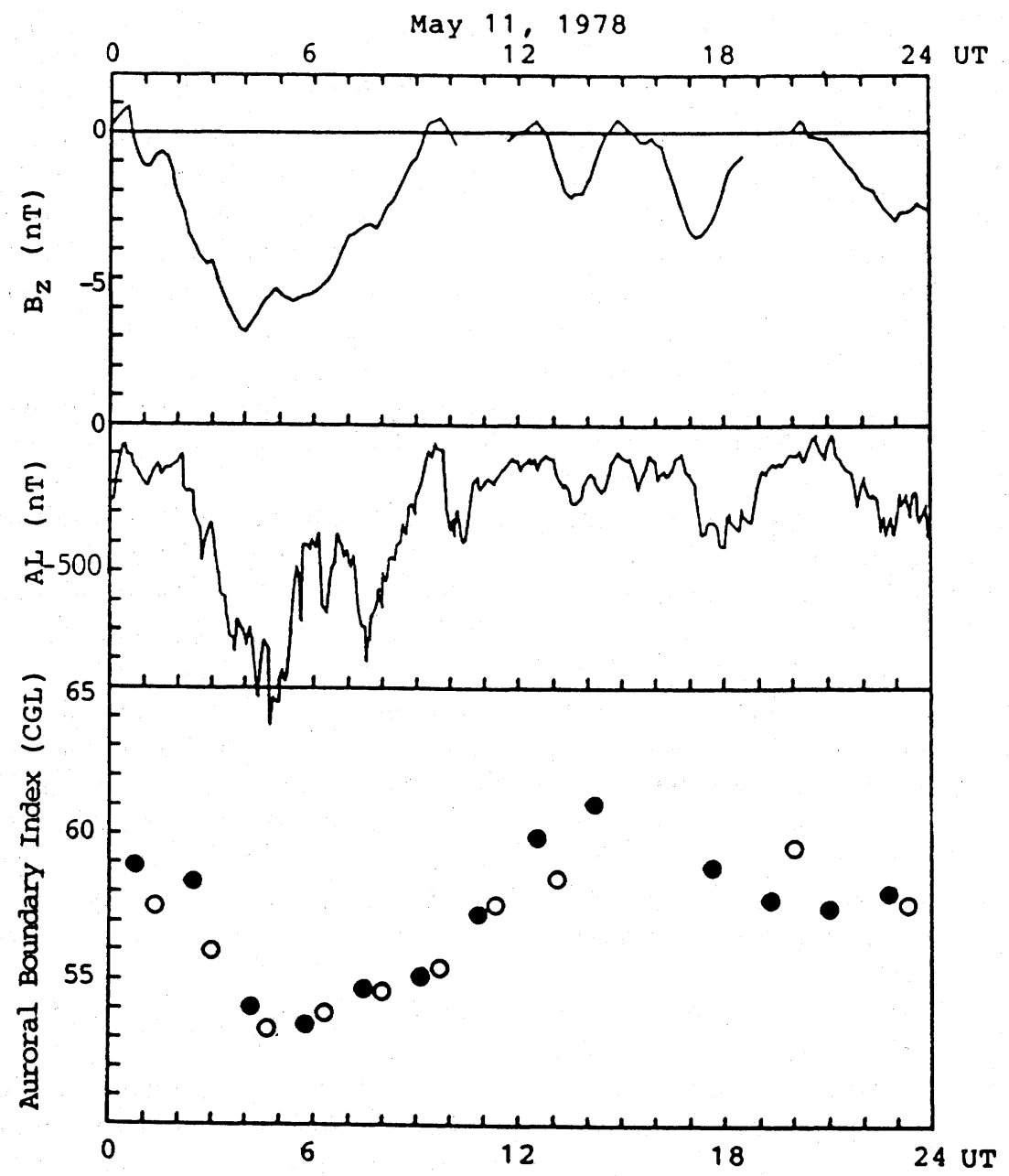

Fig. 1. Variations of the 1-hour running average of the IMF $B_{z}$ (upper panel), the $A L$ index (middle panel), and the auroral boundary index (bottom panel) on May 11, 1978. Solid and open circles indicate the auroral boundary index obtained from observations in the northern and southern hemispheres, respectively.

where the correlation coefficient is 0.76 .

\subsection{The north-south asymmetry of the auroral oval}

Since the magnetic conjugate points shift in association with variations of the tilt angle of the dipole axis, there are possibly differences between the auroral boundary indices obtained at the northern and southern ovals. FAIRFIELD and MEAD (1975) 


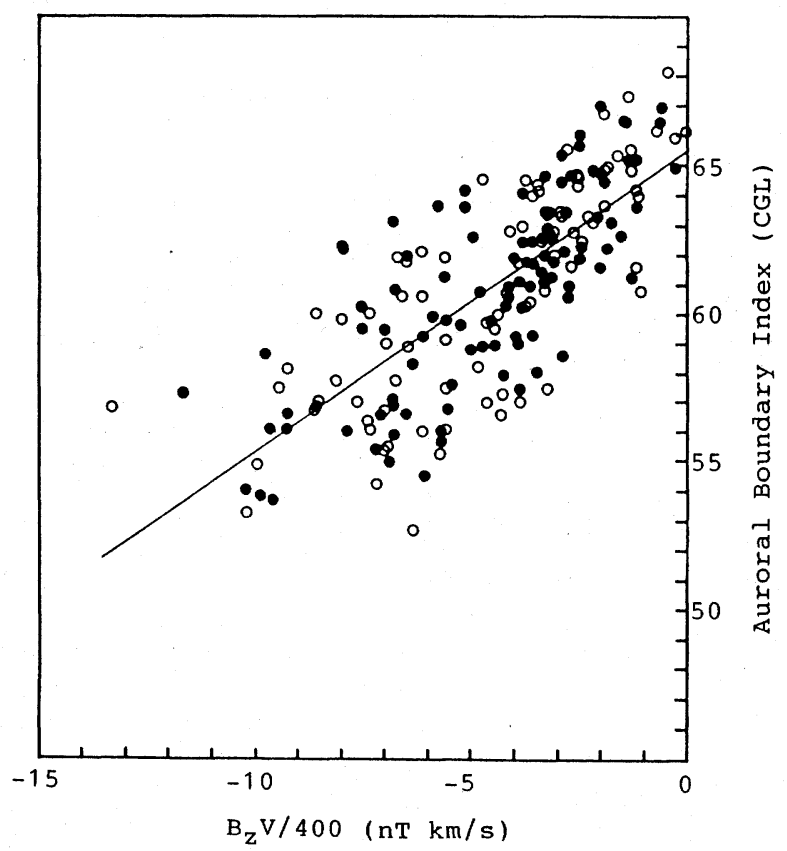

Fig. 2. The auroral boundary indices obtained within one hour of the occurrence time of the minimum $B_{z}$ are plotted as a function of the minimum values of $B_{z} V / 400$. Solid and open circles indicate the auroral boundary index obtained from the ovals in the northern and southern hemispheres, respectively. The solid line indicates a regression line.

showed that when the tilt angle is 30 or -30 degrees and $K_{\mathrm{p}} \geq 3$, a conjugate point in the summer hemisphere with a field line passing through the synchronous orbit at midnight is located about $1^{\circ}$ lower in latitude than that in the winter hemisphere. KAMIDE and WINNINGHAM (1977) also found that the equatorward boundaries of the precipitating electrons in the summer hemisphere are located about $1^{\circ}$ lower in latitude than those in the winter hemisphere. Figure 3 shows averaged values of $\Lambda-\Lambda_{\text {pred }}$ plotted as a function of the tilt angle, where $\Lambda_{\text {pred }}$ is the invariant latitude predicted from Eq. (1). Solid and open circles indicate the averages in the northern and southern hemispheres, respectively. Data are binned into four ranges, $T<-15$, $-15 \leq T<0,0 \leq T<15$, and $T \geq 15^{\circ}$. Bars attached to data points indicate the standard errors. Although the auroral boundary index in the summer hemisphere seems to be located at the lower latitude than those in the winter hemisphere, the differences are less than $1^{\circ}$, and the index is even lower in the winter hemisphere than in the summer hemisphere for the tilt angle range of $0 \leq T<15^{\circ}$. The auroral boundary index is derived from the equatorward boundary of the auroral oval in the evening sector (GUSSENHOVEN et al., 1982). Since the latitudinal difference of the magnetic 


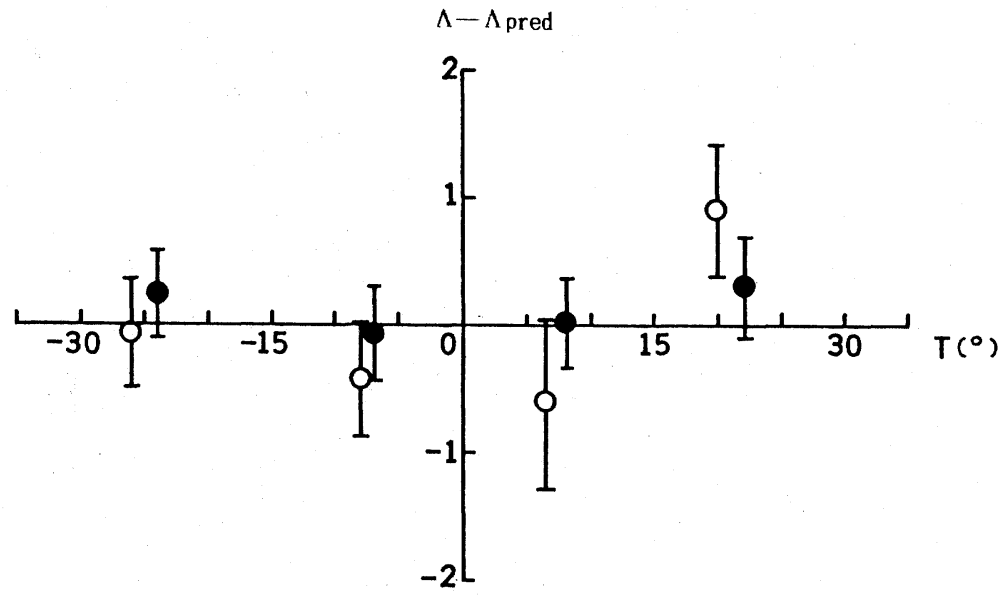

Fig. 3. Averages of differences between the auroral boundary index $(\Lambda)$ and the predictions $\left(\Lambda_{\text {pred }}\right)$ derived by using Eq. (1) are plotted as a function of the tilt angle of the dipole axis. Solid and open circles indicate the averages in the northern and southern hemispheres, respectively.

conjugate points in the evening sector is supposed to be smaller than those in the midnight sector, it is reasonable that the differences between the northern and southern indices are rather small.

It is also notable in Fig. 3 that when the tilt angle is near $0^{\circ}$ the equatorward boundaries of the auroral oval in both hemispheres seem to be located at lower latitudes than are the cases with larger absolute values of the tilt angle. This is consistent with the tilt angle dependence of the $A L$ index (AOKI, 1977; MURAYAMA $e t$ al., 1980).

\subsection{Relationship between the oval size and the polar cap potential drop}

It would be valuable to examine a quantitative relationship between the polar cap potential drop and the auroral boundary index. It is assumed that the polar cap potential drop is expressed by a linear function of the solar wind parameters using two constants, $a_{0}$ and $a_{1}$; i.e.,

$$
\Phi=a_{0}+a_{1} f
$$

where $f$ is an arbitrary function of the solar wind parameters. Using our data set, a regression function of the auroral boundary index can be substituted for $f$ in Eq. (2). For example, adopting $E(2)$ of REIFF et al. (1981), the polar cap potential drop is expressed by the auroral boundary index in the following form:

$$
\Phi=385-4.9 \Lambda \text {. }
$$


The use of the data set of DOYLE and BURKE (1983) and $B_{z} V$ as a coupling function gives

$$
\Phi=361-4.6 \Lambda
$$

Consequently it is noted that an expansion of the auroral oval by $1^{\circ}$ corresponds to an increase in the polar cap potential drop of about $5 \mathrm{kV}$.

\section{Results}

\subsection{Influences of the $B_{y}$ component of the IMF}

MURAYAMA et al. (1980) derived the influences of the IMF $B_{y}$ on the ratio of the $A L$ index to the solar wind parameters $\left(A L /\left(B_{\mathrm{s}}+0.5\right) V^{2}\right)$. In order to compare our results with Murayama et al., we introduce the following function;

$$
G=\left(\Lambda_{\mathrm{m}}-\Lambda\right) /\left(\Lambda_{\mathrm{m}}-\Lambda_{\text {pred }}\right)
$$

where $\Lambda_{\mathrm{m}}$ stands for the values of the auroral boundary index during quiet periods of auroral activity and is set at 69 CGL (MAKITA and MENG, 1984; NAKAI et al., 1986a, b), and $\Lambda_{\text {pred }}$ indicates the prediction of $\Lambda$ derived by using the Eq. (1). Then the term $\left(\Lambda_{\mathrm{m}}-\Lambda\right)$ expresses the increase in the oval size during disturbed periods, and the quantity $G$ expresses the degree of the latitudinal movement of the auroral boundary normalized by that predicted by statistics.

In Figs. 4(a), (b), and (c), $G$ values are plotted as functions of $B_{y}$ for three different seasons of the tilt angle: $T<-10,-10 \leq T<10$, and $T \geq 10^{\circ}$, respectively. When $T<-10^{\circ}, G$ values with positive $B_{y}$ is seen to be greater than those with negative $B_{y}$, and vice versa when $T \geq-10^{\circ}$. The solid lines in the figures indicate regression lines. The slopes of $B_{y}$ are $0.025,0.000$, and -0.035 in Figs. 4(a), (b), and (c), respectively. The slopes of the $B_{y}$ dependence of the $A L$ index found by MURAYAMA et al. (1980) are 0.034 and -0.028 for the tilt angle sections of $T=-28 \sim-21$ and $21 \sim 28^{\circ}$, respectively. It should be noted that the $B_{y}$ dependence of the auroral boundary index is consistent with that of the $A L$ index. Furthermore, the fact that the sensitivity of the auroral boundary index on $B_{y}$ agrees approximately with that of the $A L$ index implies that the $B_{y}$ effect on both indices comes from same sources within solar wind/magnetosphere coupling.

Since the interplanetary magnetic field lines generally follow the garden hose line, there is a strong inner correlation between $B_{x}$ and $B_{y}$. To determine which component has a strong influence on the modulation of the auroral boundary index, our data were grouped into four azimuthal sectors distinguished by the signs of $B_{x}$ and $B_{y}$. Figure 5 shows the averaged values of $G(\langle G\rangle)$ versus the azimuthal angle of the IMF $(\phi)$ measured anticlockwise from the antisunward direction viewed from the north. Starting from the top, the three panels show values of $\langle G\rangle$ with a tilt angle of $T<-10,-10 \leq T<10$, and $T \geq 10^{\circ}$. The vertical lines indicate the standard errors. When $T<-10^{\circ},\langle G\rangle$ in the negative $\phi$ is greater than unity, while $\langle G\rangle$ in the positive $\phi$ 


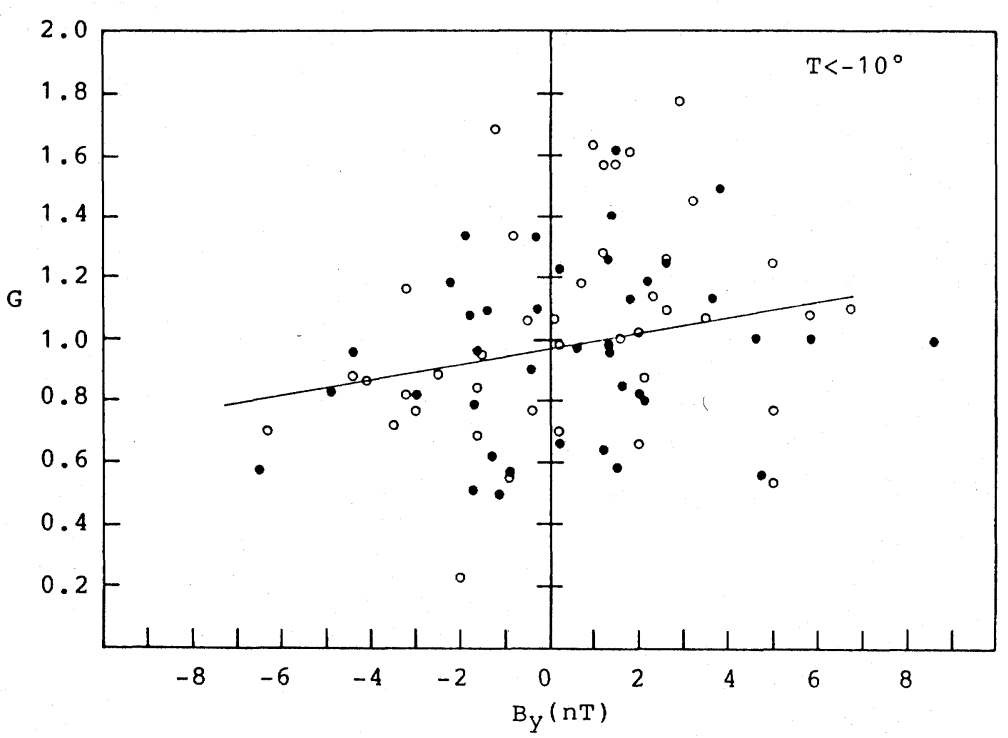

(a)

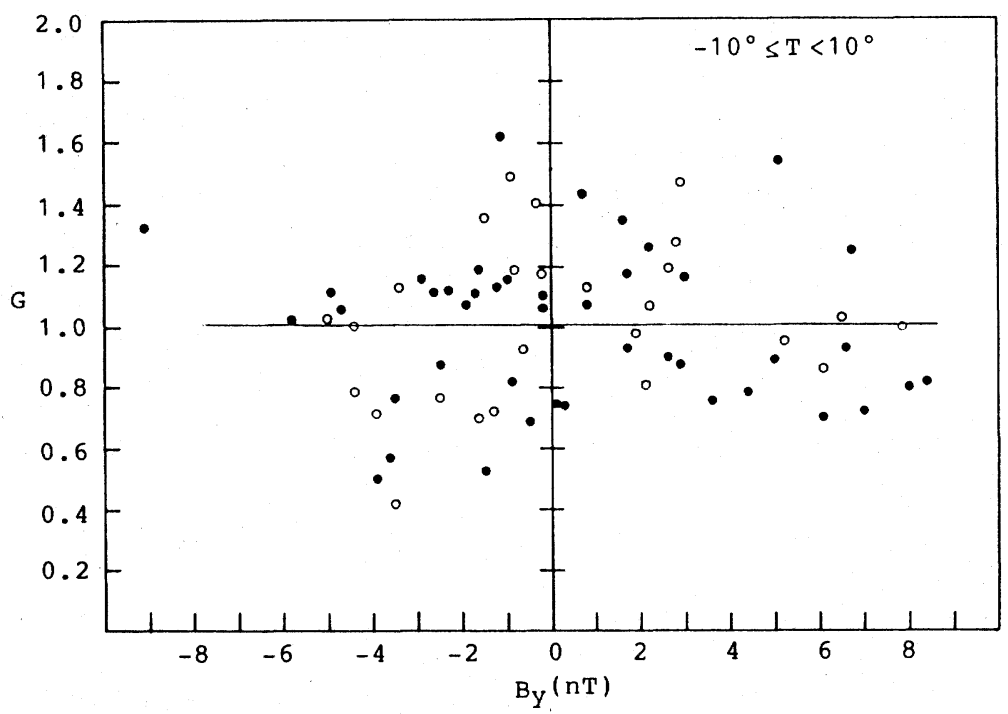

(b)

Fig. 4. (a) Values of $G=\left(69^{\circ}-\Lambda\right) /\left(69^{\circ}-\Lambda_{\text {pred }}\right)$ are plotted as a function of the IMF $B_{y}$ with the tilt angle of $T<-10^{\circ}$. Solid and open circles indicate the averages in the northern and southern hemispheres, respectively. The solid line indicates a regression line. (b) Same as (a) except for the auroral boundary index with the tilt angle of $-10 \leq T<10^{\circ}$. (c) Same as (a) except for the auroral boundary index with the tilt angle of $T \geq 10^{\circ}$. 


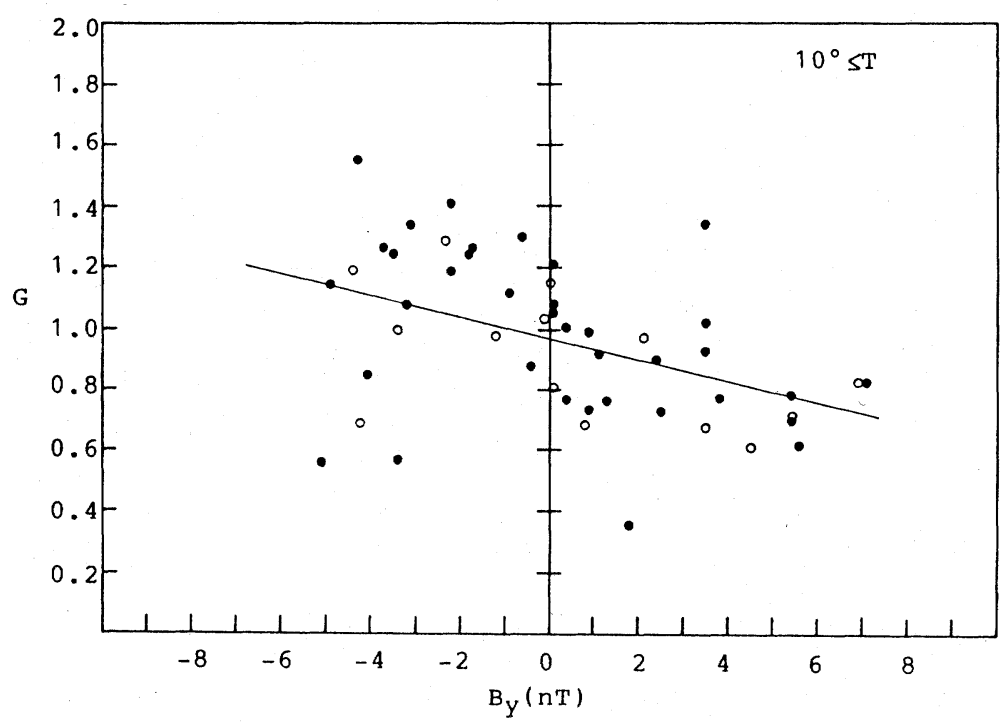

(c)

Fig. 4. (continued).

is smaller than unity, and vice versa when $T \geq 10^{\circ}$. It is significant that no statistical difference is found between the values of $\langle G\rangle$ in $\phi=-90 \sim 90^{\circ}$ and those in $\phi=90 \sim 180^{\circ}$ and $-180 \sim-90^{\circ}$. Consistent with Murayama et al. (1980), this implies that the influence of $\boldsymbol{B}_{x}$ is much less than that of $\boldsymbol{B}_{y}$.

Next, to investigate the question of whether the northern and southern ovals respond to the IMF $B_{y}$ in the same sense or in an opposite sense, our data were divided into two groups according to whether the data were obtained in the northern or southern hemispheres. In Fig. 6, the values of $G$ averaged in each bin of $B_{y}$ are plotted as a function of $B_{y}$ with circles and triangles indicating the averages with a tilt angle of $T \geq 10$ and $T<-10^{\circ}$, respectively. When $T \geq 10^{\circ},\langle G\rangle$ values for negative $B_{y}$ are larger than those for positive $B_{y}$ for both hemispheres, and vice versa when $T<-10^{\circ}$. It is evident that the oval size in both hemispheres depends on the IMF $B_{y}$ in the same sense, implying that variations of the IMF $B_{y}$ are not subject to north-south asymmetry but to a global modulation of the geomagnetic activity.

\subsection{Dependence on the direction of the transverse component}

The transverse component of the IMF, which is defined by $\boldsymbol{B}_{\mathrm{t}}{ }^{2}=\boldsymbol{B}_{y}{ }^{2}+\boldsymbol{B}_{z}{ }^{2}$ is often used in the coupling functions of the solar wind and geomagnetic activity (e.g., PERREAUlT and AKASOFU, 1978; KAN and LEE, 1979). Figures 7(a), (b), and (c) show $F$ defined by $F=\left(\Lambda_{\mathrm{m}}-\Lambda\right) /\left(\Lambda_{\mathrm{m}}-\Lambda_{\text {pred }}\right)$ as a function of $\Theta$ with tilt angles of $T<-10$, 

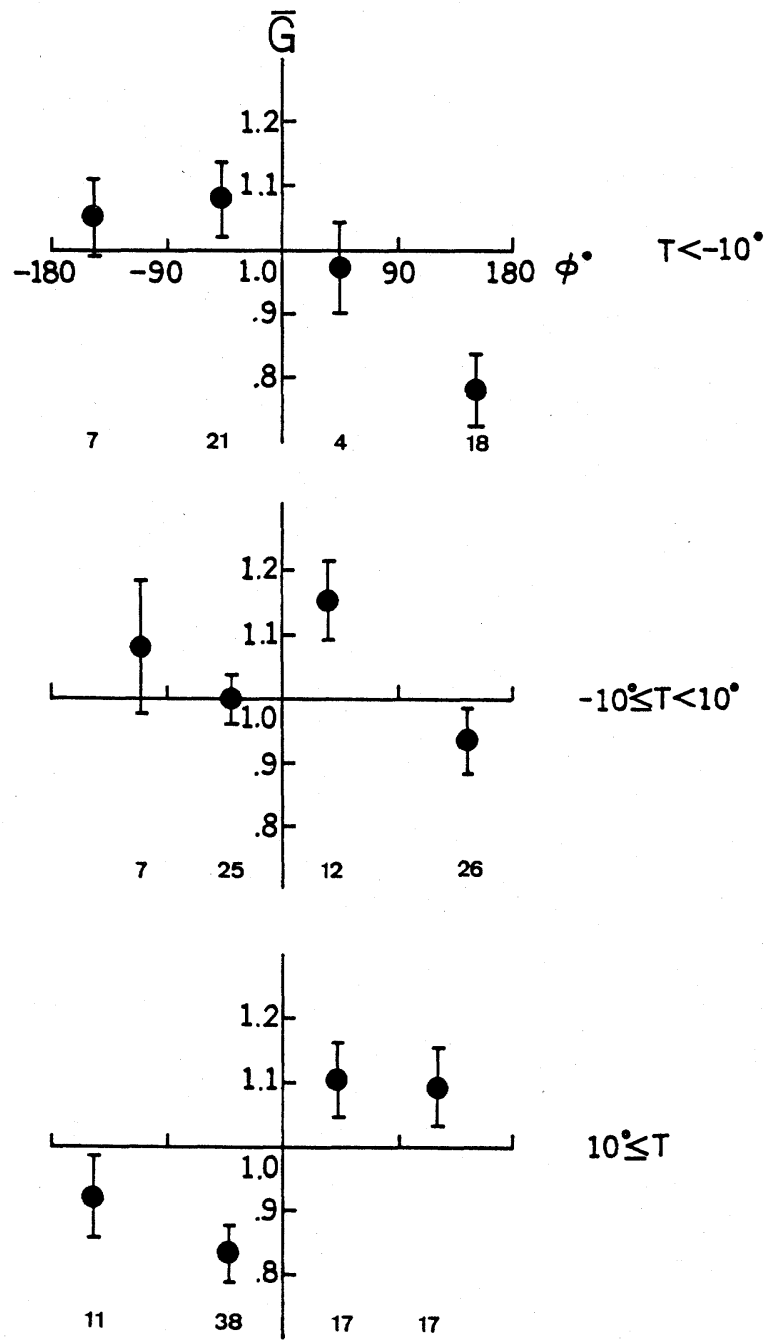

Fig. 5. Averages of $G=\left(69^{\circ}-\Lambda\right) /\left(69^{\circ}-\Lambda_{\text {pred }}\right)$ are plotted as a function of azimuthal angle measured from midnight. Bars attached to data points indicate the standard errors. The number of data in each section is shown at the bottom of the panels.

$-10 \leq T<10$, and $T \geq 10^{\circ}$, respectively, where $\Lambda_{\text {pred }}$ indicates a prediction by the regression equation

$$
\Lambda=65.4-0.78 B_{\mathrm{t}} V / 400
$$




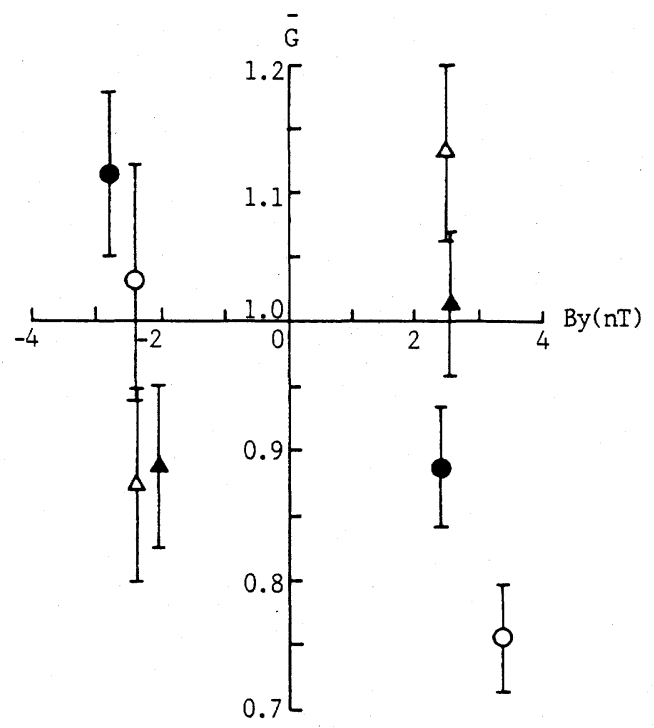

Fig. 6. Averages of $G=\left(69^{\circ}-\Lambda\right) /\left(69^{\circ}-\Lambda_{\text {pred }}\right)$ are plotted as a function of the IMF $B_{y}$. Circles and triangles indicate the averages with the tilt angle of $T \geq 10^{\circ}$ and $T<-10^{\circ}$, respectively. Solid and open symbols indicate the averages in the northern and southern hemispheres, respectively. Bars attached to each data point indicate the standard errors.

and $\Theta$ is an angle measured anticlockwise from $-z$ axis to the transverse component of the IMF viewed from the sun. The quantity $F$ indicates the normalized auroral boundary index as same as $G$ but normalized by $B_{\mathrm{t}}$.

It is evident that the values of $F$ generally decrease as absolute values of $\Theta$ increase for every section of the tilt angle. However, the axis of symmetry of data points appears to be located at $\Theta>0$ for $T<-10^{\circ}$, while it appears to be located at $\Theta<0$ for $T \geq 10^{\circ}$. A quadratic regression analysis provides us with the axes of symmetry for different tilt angles. These are at $\Theta=11.3,0.7$, and $-20.5^{\circ}$ for the tilt angle of $T<-10,-10 \leq T<10$, and $T \geq 10^{\circ}$, respectively. The regression curves are also given by solid lines in the figures. Our simple inspection for the scatter of the points in Figs. 7(a), (b), and (c) indicates that these characteristics are statistically valid. In conclusion, an alternative expression of the $B_{y}$ dependence can be given in terms of $B_{\mathrm{t}}$ and $\Theta$ such that when the tilt angle is positive (negative), the largest oval with a fixed value of $B_{\mathrm{t}}$ occurs in association with the IMF orientated westward (eastward) from the south by $10 \sim 20^{\circ}$.

\section{Summary and Discussion}

By using the auroral boundary index, the response of the oval size to variations of the transverse component of the IMF has been statistically examined. The "oval size" 


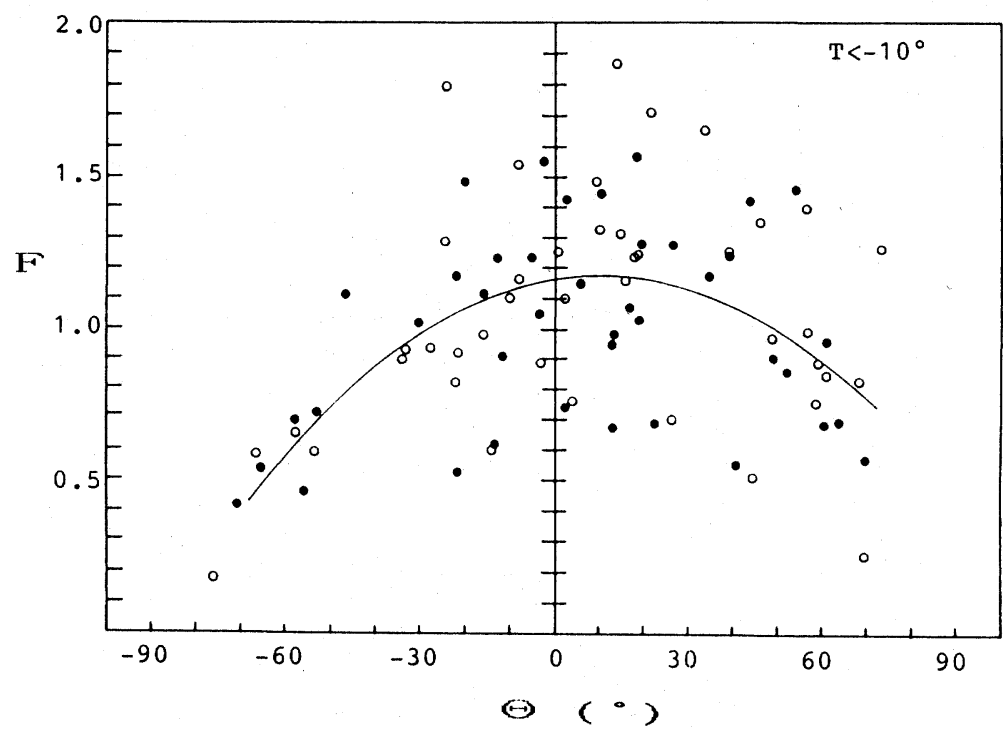

(a)

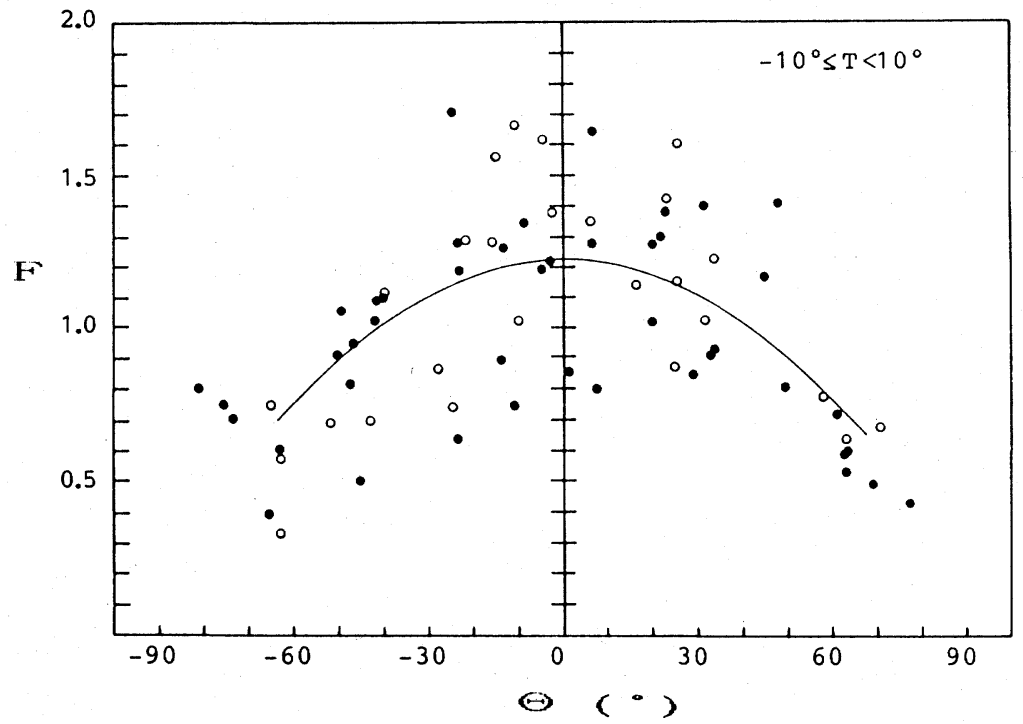

(b)

Fig. 7. (a) Values of $F=\left(69^{\circ}-\Lambda\right) /\left(69^{\circ}-\Lambda_{\text {pred }}\right)$ are plotted as a function of $\Theta$ with the tilt angle of $T<-10^{\circ}$, where $\Theta$ is an angle measured anticlockwise from the $-z$ axis to the transverse component of the IMF, viewed from the sun. Solid and open circles indicate the auroral boundary index obtained from the northern and southern hemispheres, respectively. The solid curve shows a quadratic regression line. (b) Same as (a) except for the auroral boundary index with the tilt angle of $-10 \leq T<10^{\circ}$. (c) Same as (a) except for the auroral boundary index with the tilt angle of $T \geq 10^{\circ}$. 


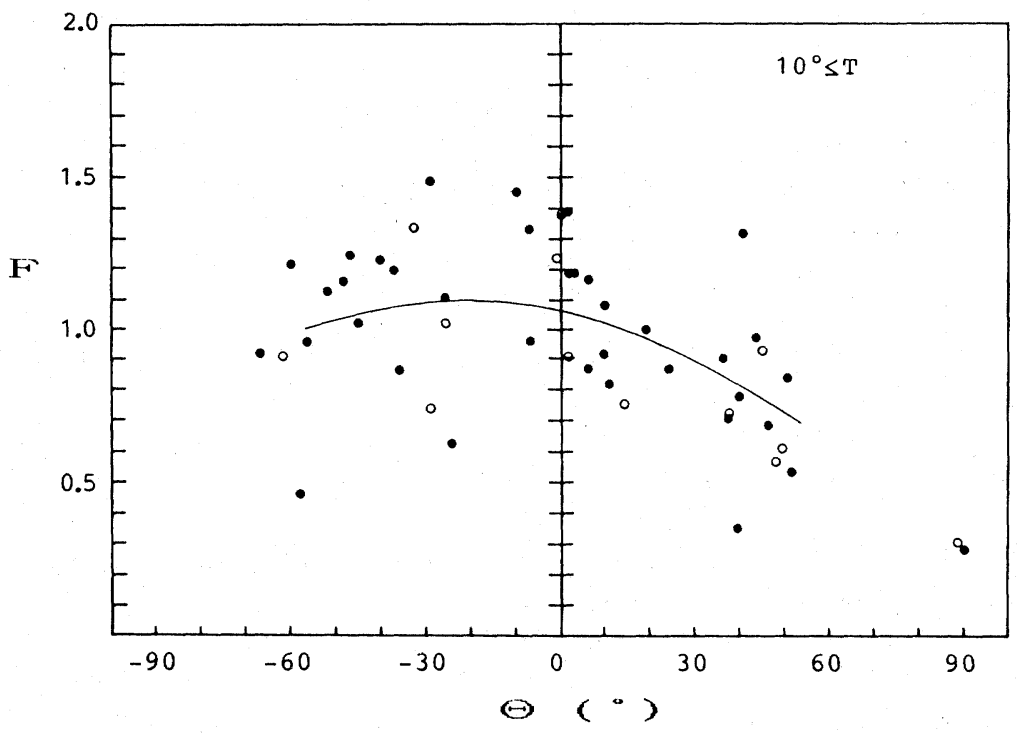

(c)

Fig. 7. (continued).

in this paper is represented by the latitude of the equatorward boundary of the auroral oval at midnight, which corresponds to the minimum $B_{z}$ in each southward excursion of the IMF. The obtained results can be summarized as follows:

(1) The oval size depends primarily on the southward component of the IMF with a correlation coefficient of 0.76 , confirming earlier studies.

(2) The expansion of the auroral oval by $1^{\circ}$ in latitude corresponds to an increase in the polar cap potential drop by about $5 \mathrm{kV}$.

(3) The size of the auroral oval with positive $B_{y}$ is larger (smaller) than that with negative $B_{y}$, when the earth's dipole tilt angle is negative (positive).

(4) The IMF $B_{x}$ component seems to be less important in controlling the oval size than the $B_{y}$ component.

(5) The $B_{y}$ effects occur in the same sense in both the northern and southern hemispheres.

(6) With a fixed magnitude of the transverse component of the IMF, the largest oval occurs in association with the IMF orientating to $\Theta=11.3,0.7$, and $-20.5^{\circ}$ when the tilt angle is in the ranges of $T<-10,-10 \leq T<10$, and $T \geq 10^{\circ}$, respectively.

The above results can be discussed in regard to two aspects of the $B_{y}$ dependence of geomagnetic activity. The first is in terms of the dayside reconnection whose efficiency has been proposed to be controlled by the transverse component of the IMF. Different authors have suggested different formulas for the dayside reconnection using parameters of the magnitude and direction of the transverse component of 
the IMF (e.g. Sonnerup, 1974; Hill, 1975; GonZalez and Mozer, 1974). However, since none of them properly took into account the effect of the tilt angle, we can not discuss our results by using existing formulas for the reconnection rate. It is interesting to note that HEELIS (1984) and CROOKER et al. (1985) suggested that a combination of the so-called antiparallel model and the dawn side preference of the dayside reconnection could explain the influence of the IMF $B_{y}$ on geomagnetic activity. However, the question of why the magnetic reconnection should occur preferably at the dawn side still remains.

The second consideration is that the $B_{y}$ dependence relates to changes in the convection pattern in the polar cap. On the basis both of satellite and ground observations, it has been revealed that the polar cap convection changes its pattern considerably in association with $B_{y}$ variations (RUSSELL, 1972; FRIIS-CHRISTENSEN and WilhJelm, 1975; MCDiarmid et al., 1979; ClAUER et al., 1984; FRIISCHRISTENSEN et al., 1985). In particular, it has been reported that when $B_{y}$ is positive (negative), ionospheric plasma flows dawnward (duskward) at the "throat" of the antisunward convection in the northern polar cusp region (HEELIS, 1984; BURCH et al., 1985). This implies that geomagnetic field lines reconnected to the IMF with positive (negative) $B_{y}$ are tugged dawnward (duskward) in the northern polar cap, and vice versa in the southern polar cap. If this is really the case, the $B_{y}$ dependence would result in a north-south asymmetry of the geomagnetic activity. This conjecture, however, is inconsistent with the finding in this paper.

The $\Theta$ dependence of the auroral size, see (6) above, can be explained in the following way to reinforce the dawnside preference of the magnetic reconnection. Figure 8 shows schematically the most preferred region of the dayside reconnection inferred from the present study. Two circles in the figure indicate the dayside
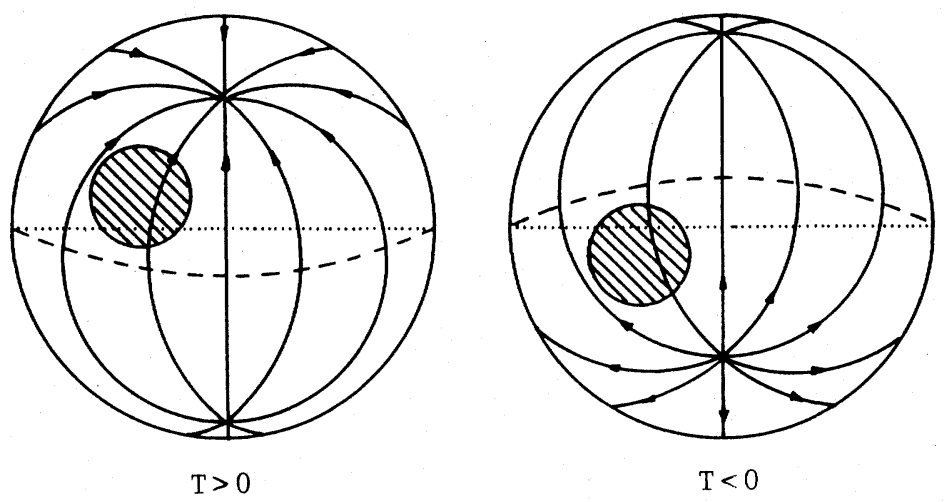

Fig. 8. Schematic views of the magnetopause with positive and negative tilt angles, shown as viewed from the sun. Shaded circles indicate the regions where the magnetic reconnection occurs most effectively. Solid curves with arrows indicate the geomagnetic field lines. Dashed and dotted lines indicate the intersections of the equatorial and ecliptic planes with the magnetopause, respectively. 
magnetopause with positive and negative tilt angle viewed from the sun. Solid curves with arrows indicate the geomagnetic field lines. Dashed and dotted lines indicate the intersections of the equatorial and ecliptic plane with the magnetopause, respectively. Since the magnetic reconnection is supposed to depend partially on the magnitude of the sheath field compression in front of the magnetopause, the reconnection usually occurs in the hemisphere where the subsolar point is located. When the tilt angle is positive, the dominant reconnection region is located in the northern hemisphere, since the subsolar point is located in the northern hemisphere. On the other hand, for the negative tilt angle, the dominant reconnection region would be located in the southern hemisphere. Theoretical and numerical studies of the magnetic reconnection suggest that the reconnection rate becomes its maximum when two colliding magnetic fields are antiparallel (e.g., HILL, 1975; HoSHINO and NISHIDA, 1983). Combining (6) in our result and this feature, we infer that the reconnection should take place most effectively on the dawn side rather than around the noon-midnight meridian, as shown by shaded circles in Fig. 8. Although the region where the geomagnetic field is antiparallel to the sheath field extends from the dayside cusp region to the flank of the magnetopause (see LUHMANN et al., 1984), the assumption that the reconnection rate depends partially on the magnitude of the field compression leads to the conclusion that the reconnection generally occurs at low latitudes. Note, however, that this view does not deny the existence of reconnection at high latitudes.

Several hypotheses have been suggested to explain the dawnside preference of the magnetic reconnection. The first follows the observations in which the stagnation point of the solar wind was displaced dawnward from the subsolar point. However it was shown theoretically by RUSSELL et al. (1981) and statistically by CROOKER et al. (1984) that such a displacement occurs only when the solar wind Alfvén Mach number is extremely low, i.e., $M a<4$ (CROOKER et al., 1985). The second interpretation is that magnetic reconnection will be favored in the region where the vectors of the IMF and of the earth's magnetic field are purely antiparallel (HEELIS, 1984). This indicates that the IMF $B_{x}$ plays a more important role in the dayside reconnection than does $B_{y}$. However, as CROOKER et al. (1985) pointed out, this hypothesis contradicts MURAYAMA et al. (1980) and (4) above that geomagnetic activity does not depend very much on variations in the IMF $B_{x}$ (Murayama, private communication, 1986).

These two hypotheses agree with each other on the standpoint that the magnetosheath field configuration in front of the magnetopause is responsible for the dawnside preference. Another possible aspect is to attribute the cause of the dawnside preference to the inside of the magnetosphere. The loss of the magnetic flux tugged away from the front closed region to the tail lobe is compensated for by the return flux from the tail. The dayside magnetopause thereby moves inward or outward following the variation of the reconnection rate and reaches a new equilibrium position as soon as the balance is achieved (CORONITI and KENNEL, 1973). We can speculate that the return flux from the tail will work as positive feedback to control the dayside reconnection rate. Circumstantial evidence for this speculation is found in the observation that at the stagnation point of the dayside magnetopause the strength of the magnetosheath field is proportional to the geometric mean of the stagnation field 
strength and the transverse component of the IMF (CROOKER et al., 1982). Ground magnetometer and radar observations indicate that the potential and convection pattern in the northern polar cap are rotated clockwise by $15^{\circ} \sim 45^{\circ}$ with respect to the noon-midnight meridian (EVANS et al., 1980; KAMIDE et al., 1981), hence the return flux mapped from the ionospheric sunward convection on the equatorial plane would convect from $2100 \sim 2300$ MLT to $0900 \sim 1100$ MLT. Therefore, since the positive feedback from the return current is assumed to be most effective at the dawnside region to the magnetopause, it creates the dawnside preference of the magnetic reconnection. Theoretical or numerical studies on the dayside reconnection model including the return flux from the tail are required in the near future.

Since the oval size is proportional to the polar cap potential drop, values of $F$ shown in Fig. 7 indicate a $\Theta$-dependence of the magnitude of the electric field induced by the dayside reconnection. Normalizing at $\Theta=0$, values of $F$ averaged in each $\Theta$ 's bin of $15^{\circ}$ are shown in Fig. 9 by solid circles for the tilt angle section from $-10^{\circ}$ to $10^{\circ}$, where $E / E_{0}$ stands for $\langle F\rangle / F(\Theta=0)$. Two solid curves show the theoretical predictions by HILL (1975);

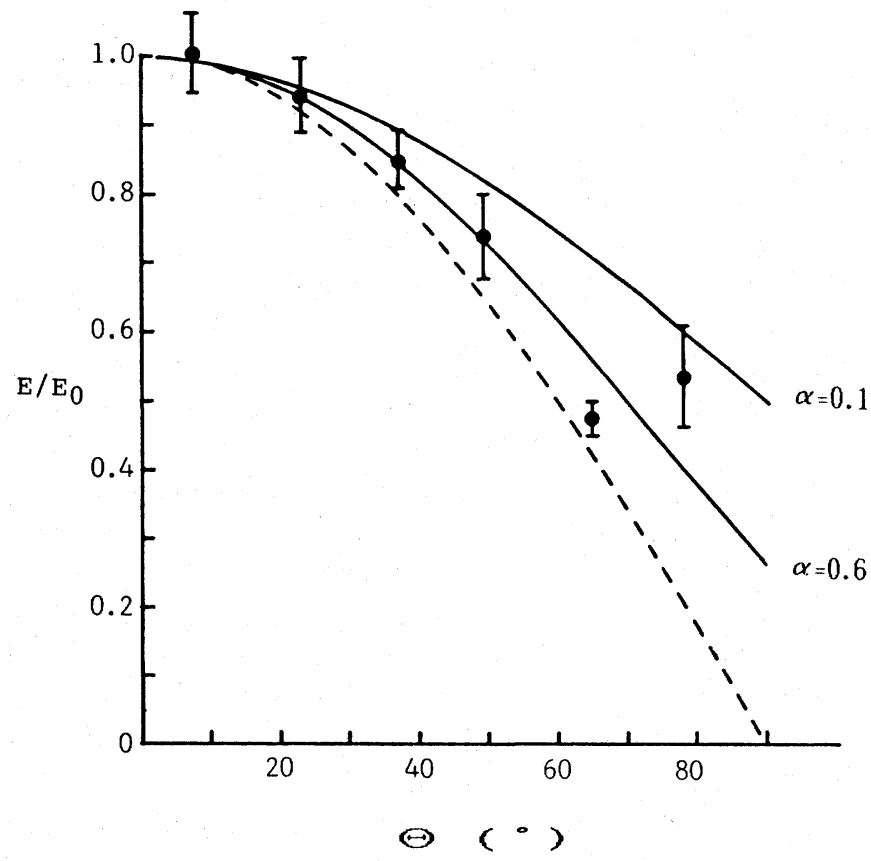

Fig. 9. $\Theta$ dependence of the electric field induced by the dayside magnetic reconnection $\left(E / E_{0}\right)$. Data points are obtained by averaging normalized values of $F=\left(69^{\circ}-\Lambda\right) /\left(69^{\circ}-\Lambda_{\text {pred }}\right)$. Bars attached to each data point indicate the standard errors. Solid curves show theoretical predictions derived by using HILL's (1975) equation with $\alpha=1.0$ and 0.6 . The dashed curve is predicted from the half-wave rectifier model. 


$$
E / E_{0}=(\alpha-\cos \theta)^{2} /\left(1+\alpha^{2}-2 \alpha \cos \theta\right)
$$

where $\theta=\pi-|\Theta|$, and $\alpha$ is the ratio of the magnitude of the magnetosheath field to that of the geomagnetic field at both sides of the separator. The two curves are for $\alpha=1.0$ and 0.6 . The dashed line represents the prediction from the half-wave rectifier model, i.e., $E / E_{0}=\cos \Theta$. It is noted that the data points are distributed slightly above the prediction by the half-wave rectifier model (HAKAMADA et al., 1980) and generally agree with HILL (1975) for $\alpha=0.6$. This confirms the earlier result that Eq. (7) can predict the magnitude of the polar cap potential drop reasonably well (REIFF et al., 1981; WYGANT et al., 1983; DOYLE and BURKE, 1983).

The author is indebted to Y. Kamide for his kind guidance and valuable discussions. Data of the interplanetary magnetic field and the solar wind from IMP-J were provided by N. F. Ness through the WDC-A for Rockets and Satellites in GSFC/NASA. K. Maezawa assisted the author in rearranging the interplanetary magnetic field records. The present study was sponsored in part by the Ministry of Education in Japan under Grant-in-Aids for Scientific Research 61916030.

\section{REFERENCES}

AOKı, T., Influence of the dipole tilt angle on the development of auroral electrojets, J. Geomag. Geoelectr., 29, 441-453, 1977.

Burch, J. L., P. H. Reiff, J. D. Menietti, R. A. Heelis, W. B. Hanson, S. D. Shawhan, E. G. Shelley, M. Sugiura, D. R. Weimer, and J. D. Winningham, IMF $B_{y}$-dependent plasma flow and Birkeland currents in the dayside magnetosphere 1. Dynamics Explorer observations, J. Geophys. Res., 90, 1577-1593, 1985.

Clauer, C. R., P. M. Banks, A. Q. Smith, E. Frils-Christensen, S. Vennerstr $\emptyset$ M, V. B. Wickwar, J. D. Kelly, and J. DouPNiK, Observation of interplanetary magnetic field and of ionospheric plasma convection in the vicinity of the dayside polar cleft, Geophys. Res. Lett., 1, 891-894, 1984.

Coroniti, F. V. and C. F. KENnEL, Can the ionosphere regulate magnetospheric convection ?, J. Geophys. Res., 78, 2837-2851, 1973.

Crooker, N. U., G. L. Siscoe, P. R. Mullen, C. T. Russell, and E. J. Smith, Magnetic field compression at the dayside magnetopause, J. Geophys. Res., 87, 10407-10412, 1982.

Crooker, N. U., G. L. Siscoe, T. E. Eastman, L. A. Frank, and R. D. ZwickL, Large scale flow in the dayside magnetosheath, J. Geophys. Res., 89, 9711-9719, 1984.

Crooker, N. U., J. G. Luhmann, J. R. Spreiter, and S. S. Stahara, Magnetopause merging site asymmetries, J. Geophys. Res., 90, 341-346, 1985.

Doyle, M. A. and W. J. Burke, S3-2 measurements of the polar cap potential, J. Geophys. Res., 88, 9125-9133, 1983.

Evans, J. V., J. M. Holt, W. L. Oliver, and R. H. WaRd, Millstone Hill incoherent scatter observations of auroral convection over $60<\Lambda<75$.: Initial results, J. Geophys. Res., 85, 41-54, 1980.

FAIRFIELD, D. H. and C. D. MEAD, Magnetospheric mapping with a quantitative geomagnetic field model, J. Geophys. Res., 80, 535-542, 1975.

Fris-Christensen, E. and J. WilhJelm, Polar cap currents for different directions of the interplanetary magnetic field in the $y$-z plane, J. Geophys. Res., 80, 1248-1260, 1975.

Fris-Christensen, E., K. Lassen, J. Wilhjelm, J. M. Wilcox, W. Gonzalez, and D. S. Cotburn, Critical component of the interplanetary magnetic field responsible for large geomagnetic effects in the polar cap, J. Geophys. Res., 77, 3371-3376, 1972. 
Friss-Christensen, E., Y. Kamide, A. D. Richmond, and S. Matsushita, Interplanetary magnetic field control of high-latitude electric fields and currents determined from Greenland magnetometer data, $J$. Geophys. Res., 90, 1325-1338, 1985.

Gonzalez, W. D. and F. S. Mozer, A quantitative model for the potential resulting from reconnection with an arbitrary interplanetary magnetic field, J. Geophys. Res., 79, 4186-4194, 1974.

Gussenhoven, M. S., D. A. Hardy, N. Heinemann, and E. Holeman, 1978 diffuse auroral boundaries and a derived auroral boundary index, $A F G L-T R-82-C 398$, Air Force Geophys. Lab., Hanscom Air Force Base, Bedford, Mass., Dec. 1982.

Gussenhoven, M. S., D. A. Hardy, and N. Heinemann, Systematics of the equatorward diffuse auroral boundary, J. Geophys. Res., 88, 5692-5708, 1983.

HAKAMADA, K., T. AOKI, and T. MURAYAMA, Influence of the east-west component of the interplanetary magnetic field on the intensity of the auroral electrojet, Planet. Space Sci., 28, 29-39, 1980.

Hardy, D. A., W. J. Burke, M. S. Gussenhoven, N. Heinemann, and E. Holeman, DMSP/F2 electron observations of equatorward auroral boundaries and their relationship to the solar wind velocity and the north-south component of the interplanetary magnetic field, J. Geophys. Res., 86, 9961-9974, 1981 .

HEELIS, R. A., The effect of interplanetary magnetic field orientation on dayside high-latitude ionospheric convection, J. Geophys. Res., 89, 2873-2880, 1984.

HePpner, J. P., Polar-cap electric field distributions related to the interplanetary magnetic field direction, J. Geophys. Res., 77, 4877-4887, 1972.

HiLl, T. W., Magnetic merging in a collisionless plasma, J. Geophys. Res., 80, 4689-4699, 1975.

Holzworth, R. H. and C.-I. Meng, Mathematical representation of the auroral oval, Geophys. Res. Lett., 2, 377-380, 1975.

Hoshino, M. and A. Nishida, Numerical Simulation of the Dayside Reconnection, J. Geophys. Res., 88, 6926-6936, 1983.

KAmide, Y. and J. D. Winningham, A statistical study of the instantaneous nightside auroral oval: The equatorward boundary of electron precipitation as observed by the ISIS 1 and 2 satellites, J. Geophys. Res., 82, 5573-5588, 1977.

Kamide, Y., A. D. Richmond, and S. MATSUShita, Estimation of ionospheric electric fields, ionospheric currents, and field-aligned currents from ground magnetic records, J. Geophys. Res., 86, 801-813, 1981.

KAN, J. R. and L. C. LEE, Energy coupling function and solar wind-magnetosphere dynamo, Geophys. Res. Lett., 6, 577-580, 1979.

Luhmann, J. G., R. J. Walker, C. T. Russell, N. U. Crooker, J. R. Spreiter, and S. S. Stahara, Patterns of magnetic field merging sites on the magnetopause, J. Geophys. Res., 89, 1739-1742, 1984.

Makita, K. and C.-I. MenG, Average electron precipitation patterns and visual auroral characteristics during quiescence, J. Geophys. Res., 89, 2861-2872, 1984.

Matsushita, S. and W.-Y. Xu, Equivalent ionospheric current systems representing IMF sector effects on the polar geomagnetic field, Planet. Space Sci., 30, 641-656, 1982.

McDiarmid, I. B., J. R. Burrows, and M. D. Wilson, Large-scale magnetic field perturbations and particle measurements at $1400 \mathrm{~km}$ on the dayside, J. Geophys. Res., 84, 1431-1441, 1979.

Murayama, T., T. AOKI, H. NAKAI, and K. HAKAMADA, Empirical formula to relate the auroral electrojet intensity with interplanetary parameters, Planet. Space Sci., 28, 803-813, 1980.

NAKAI, H. and Y. KAmIDE, Response of nightside auroral oval boundaries to the interplanetary magnetic field, J. Geophys. Res., 88, 4005-4014, 1983.

NAKAI, H., Y. Kamide, D. A. HARDY, and M. S. GuSsenhoven, Time scales of expansion and contraction of the auroral oval, J. Geophys. Res., 91, 4437-4450, 1986a.

Nakai, H., Y. Kamide, D. A. Hardy, and M. S. Gussenhoven, The dynamics of the equatorward boundary of the auroral oval, in Solar Wind-Magnetosphere Coupling, edited by Y. Kamide and J. A. Slavin, pp. 633-641, Terrapub/Reidel, Tokyo, 1986b.

Perreault, P. and S.-I. Akasofu, A study of geomagnetic storms, Geophys. J. Astron. Soc., 54, 547-573, 1978. 
PIKE, C. P. and B, S. DANDEKAR, Evening sector auroral oval dynamics from DMSP photographs, $J$. Geophys. Res., 84, 3389-3402, 1979.

Potemra, T. A. and N. A. Saflekos, Birkland currents and the interplanetary magnetic field, in Magnetospheric Boundary Layers, edited by B. Battrick, pp. 193-198, Eur. Space Agency Spec. Publ., ESA SP-148, 1979.

ReifF, P. H., R. W. Spiro, and T. W. HiLl, Dependence of polar cap potential drop on interplanetary parameters, J. Geophys. Res., 86, 7639-7648, 1981.

Russell, C. T., The configuration of the magnetosphere, in Critical Problems of Magnetospheric Physics, edited by E. R. Dyer, pp. 1-16, National Academy of Sciences, Washington, D.C., 1972.

Russell, C. T., H.-C. Zhuang, R. J. WAlker, and N. U. Crooker, A note on the location of the stagnation point in the magnetosheath flow, Geophys. Res. Lett., 8, 984-986, 1981.

Sonnerup, B. U. Ö., Magnetopause reconnection rate, J. Geophys. Res., 79, 1546-1549, 1974.

Wygant, J. R., R. B. TorberT, and F. S. Bozer, Comparison of S3-3 polar cap potential drops with the interplanetary magnetic field and models of magnetopause reconnection, J. Geophys. Res., 88, 5727-5735, 1983. 